\title{
LA INDUSTRIA AGROALIMENTARIA EN ANDALUCÍA
}

\author{
Aarón José Leal Borrell \\ Estudiante de la Licenciatura en Geografía. Universidad de Alicante (España) \\ Correo electrónico: leal_cast@hotmail.com
}

Recibido: 20 de abril de 2011. Devuelto para revisión: 12 de mayo de 2011.

Aceptado: 20 de junio de 2011

\begin{abstract}
RESUMEN
Andalucía ha tenido y sigue teniendo un papel muy destacado dentro del sector agrario español. Una de sus ramas con más proyección y perspectiva de futuro es la agroalimentaria debido a los cambios de tendencia de los consumidores y a las nuevas demandas de productos más innovadores, de más calidad y de más rápida preparación. El presente trabajo tiene la intención de servir como introducción a un sector estratégico que no hay que dejar de lado ya que es capaz de mover cantidades ingentes de dinero y de generar numerosos puestos de trabajo. A pesar de ello, la situación económica actual no le es ajena por lo que hay que buscar nuevas formas de agrupación más competitivas y nuevos mercados que no estén saturados.
\end{abstract}

Palabras clave: Agroindustria, Andalucía, Cooperativas, Exportaciones.

\begin{abstract}
Andalucía has had and still has a very important role in the Spanish agrarian sector. One of its branches with a more relevant perspective of future is the agrifood due to the consumers changing tastes and to the new demands of more sophisticated products (o better quality and faster preparation). The present work is meant to be an introduction to a strategic sector worth considering because it is able to move huge amounts of money and to generate many jobs. In spite of this, the actual economic situation is present so there is a need to look for more competitive groupings and new and not saturated markets.
\end{abstract}

Key words: Agribusiness, Andalucía, Cooperatives, Exports.

\section{INTRODUCCIÓN}

Andalucía está situada al sur de la Península Ibérica, entre $\operatorname{los} 36^{\circ}$ y los $38^{\circ} \mathrm{N}$, lo que la convierte en el punto más meridional del continente europeo. Su situación geográfica hace de esta comunidad un lugar idóneo para la implantación de cultivos y demás actividades primarias, base fundamental de obtención de materias primas para el desarrollo de la industria agroalimentaria, centro de este estudio.

La agroindustria es un sistema de elaboración que implica la combinación de dos procesos productivos, el agrícola y el industrial, para transformar de manera rentable los productos provenientes del campo. Se define como un conjunto de procesos de 
transformación aplicados a materias primas de origen agropecuario, pesquero y forestal, que abarca desde su primera agregación de valor hasta las empresas de distribución.

En Andalucía, la agroindustria es un sector de importancia capital y así se recoge en el Plan Andaluz de Desarrollo Industrial (PADI) 2008-2013, en el que se define a éste como uno de los sectores estratégicos de la economía andaluza, junto con otros 6 sectores de carácter industrial y tecnológico. Además, "se le puede considerar el motor económico de las zonas rurales ya que actúa de vínculo entre la producción agraria, el territorio y el medio rural" (Consejería de Agricultura y Pesca, 2006).

Dada la importancia del sector en esta comunidad he creído conveniente la realización de este estudio en el que se persiguen unos objetivos no demasiado profundos pero en el que se intentará reflejar de manera fiel la situación actual del sector contemplando los aspectos más representativos y destacados del mismo.

Para la realización de este estudio se harán referencia en varias ocasiones a datos cuantitativos extraídos de la serie de Anuarios Estadísticos de la Junta de Andalucía y del propio Instituto Nacional de Estadística. Además, también se ha hecho uso de otras fuentes estadísticas de empresas privadas como DATACOMEX.

Según el documento "El complejo agroalimentario andaluz. Presente y futuro", editado por el Instituto de Estudios Cajamar en 2005, la agroindustria andaluza se enfrenta a un contexto de cambio debido a las modificaciones que se están produciendo en el marco institucional con la ampliación de la Unión Europea y el proceso de liberalización comercial impulsado por organismos económicos y comerciales europeos. Además, los constantes cambios en el mercado con nuevas demandas del consumidor, que desean productos más sanos, más nutritivos y más fáciles de preparar hacen que este sector esté en perpetua evolución y adaptación.

Como se verá más adelante, Andalucía es una de las principales regiones agrarias europeas, por encima de países de la Unión Europea como Finlandia, Bélgica o Portugal. Asimismo, para productos como el aceite de oliva y ciertas hortalizas es un referente a nivel mundial, aspecto también potenciado por su situación entre el Mar Mediterráneo y el Océano Atlántico.

Para acabar con este apartado es interesante también hacer una pequeña referencia al contexto socioeconómico de la región, que ha sufrido una fuerte evolución en los últimos años. Como se cita en el Plan Estratégico para la Agroindustria Andaluza. Horizonte 2013, existen una serie de características que definen el marco actual en el que se mueve este sector. Por un lado existe una fuerte globalización de la economía, que supone más competencia entre países, más competencia por las materias primas, aunque también más posibilidades de mercado. También ha variado el perfil del consumidor, que busca más salud, comodidad, calidad y productos más innovadores. Además, los nuevos consumidores también se preocupan por la sostenibilidad y la seguridad de los alimentos. Por otro lado, en lo referente a las agroindustrias, hay que destacar los procesos de concentración de la distribución, la innovación y las inversiones y la atomización de las empresas. 
Asimismo, hay que tener en cuenta que el marco económico nacional y mundial se encuentra inmerso en una profunda crisis agravada por la globalización de la economía y la creciente interdependencia entre estados y empresas. No obstante, esta situación coyuntural está propiciando mayor innovación y la expansión del sector hacia el continente asiático en busca de nuevos mercados emergentes que no estén saturados de productos agroalimentarios, lo que abre nuevas posibilidades de crecimiento del sector.

\section{CARACTERÍSTICAS GENERALES DE LA AGROINDUSTRIA ANDALUZA}

La industria agroalimentaria andaluza representa casi un 3\% del PIB de la comunidad. Dos aspectos importantes de este sector en la región son el equilibrio económico y su grado de exportación. El primero de ellos se debe a que la mayor parte de estas empresas se encuentran en zonas rurales, aunque ello también conlleva un alto grado de atomización y dispersión de las mismas. Del segundo aspecto cabe destacar su evolución favorable desde mediados de los años noventa del siglo pasado.

A través de los anuarios del Instituto Estadístico de Andalucía se ha obtenido una tabla que refleja las principales características de la industria agroalimentaria en esta comunidad en la última década.

\section{Cuadro 1. Características generales de la industria agroalimentaria en Andalucía (2000-2008).}

\begin{tabular}{|c|c|c|c|c|c|}
\cline { 2 - 6 } \multicolumn{1}{c|}{} & $\mathbf{2 0 0 0}$ & $\mathbf{2 0 0 2}$ & $\mathbf{2 0 0 4}$ & $\mathbf{2 0 0 6}$ & $\mathbf{2 0 0 8}$ \\
\hline Personas ocupadas $^{*}$ & 53.856 & 51.750 & 50.106 & 52.516 & 52.505 \\
\hline Ingresos de explotación $^{*}$ & 9.071 .596 & 11.375 .336 & 12.319 .291 & 13.257 .569 & 14.723 .330 \\
\hline Gastos de explotación & $* 4.415 .269$ & 10.789 .797 & 11.852 .789 & 12.652 .006 & 14.294 .629 \\
\hline $\begin{array}{c}\text { Inversión realizada en } \\
\text { activos materiales }\end{array}$ & 226.692 & 487.953 & 544.076 & 644.560 & 633.271 \\
\hline
\end{tabular}

Fuente: IEA. Encuesta Industrial Anual de Empresas. Resultados para Andalucía. Elaboración propia.

* Datos en millones de $€$

De ella se pueden extraer varias conclusiones a partir de los datos observados y también a partir de las relaciones que se pueden realizar entre ellos. En primer lugar, se observa un descenso general de la población ocupada en este sector desde el año 2000 hasta el año 2008. En segundo lugar se ve como ha aumentado en gran medida tanto los ingresos y los gastos por explotación como las inversiones realizadas en activos materiales. Si ponemos en relación varios aspectos se puede destacar que los ingresos y los gastos por explotación van prácticamente a la par, es decir, a medida que aumentan los ingresos, los agricultores pueden invertir más, tanto en activos materiales como en bienes duraderos.

En un análisis más detallado se puede separar el cuadro en dos etapas: del año 2000 al 2005, y desde este último hasta el año 2008. En esta primera etapa hay que destacar un gran descenso de la población activa además de un gran aumento de los ingresos y gastos por explotación. No obstante, lo que más llama la atención es el gran aumento de 
la inversión en bienes materiales. En esta segunda etapa se puede ver como se recupera algo la población activa, aunque sin llegar a los niveles del año 2000. También se ve como suben los ingresos y gastos por explotación y las inversiones, aunque en menor medida. Este hecho puede estar producido por la crisis socioeconómica que vivimos en la actualidad y de la que ya en el año 2005 empezaban a notarse sus primeras consecuencias. Igualmente, el aumento del paro debido a esta crisis puede haber provocado que una parte de la población busque empleo en este sector económico que, desde hace algunas décadas, está dominado por la población inmigrante.

Como se puede ver en estas tablas elaboradas a partir de datos extraídos de la expresa DATACOMEX, Andalucía tiene una tasa de cobertura de los alimentos consumidos en su territorio mucho mayor que el conjunto de España, es decir, que no necesita importar tantos alimentos para satisfacer las necesidades de consumo de su mercado interno. Además, esta afirmación viene corroborada por la Figura 2 en la que apreciamos que las exportaciones de alimentos de Andalucía superan en varios cientos de millones de euros a sus importaciones debido sobre todo a que la mayoría de las empresas andaluzas están orientadas hacia mercados del norte y del centro de Europa. No obstante, hay que aclarar que estos datos no se refieren solo a alimentos del sector agroalimentario sino a todos los alimentos importados y exportados.

Otro aspecto a destacar en estas tablas es el descenso que se ha dejado sentir en todas las actividades productivas y económicas desde mediados de 2008 como consecuencia de la coyuntura económica que atraviesan todos los países desarrollados.

Figura 1. Tasas de Cobertura de alimentos en España y Andalucía (1995-2010).

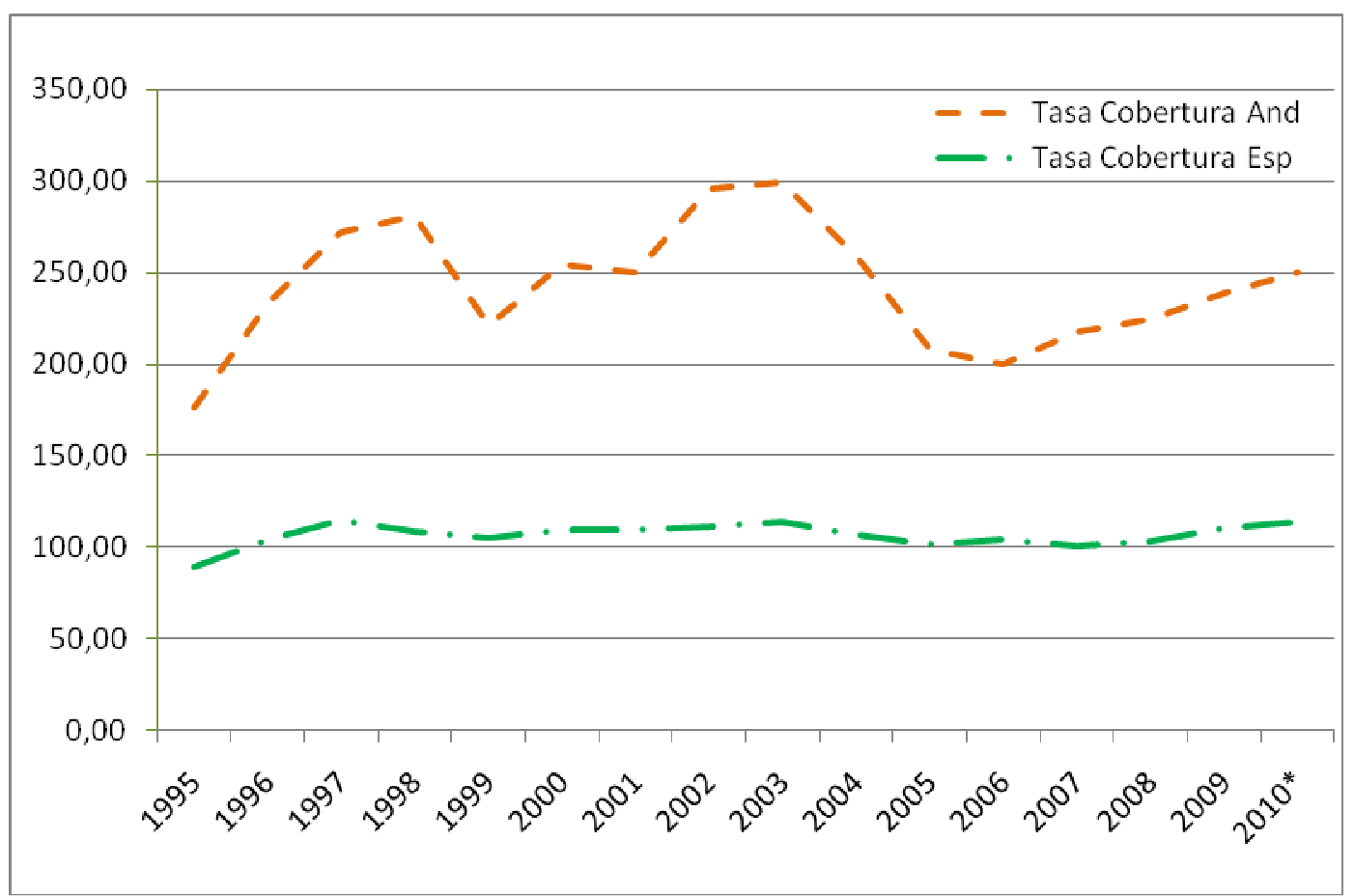

Fuente: DATACOMEX. Elaboración propia. 


\section{Figura 2. Exportación-Importación de todos los alimentos en Andalucía en el periodo 1995 - 2010 (en millones de Euros).}

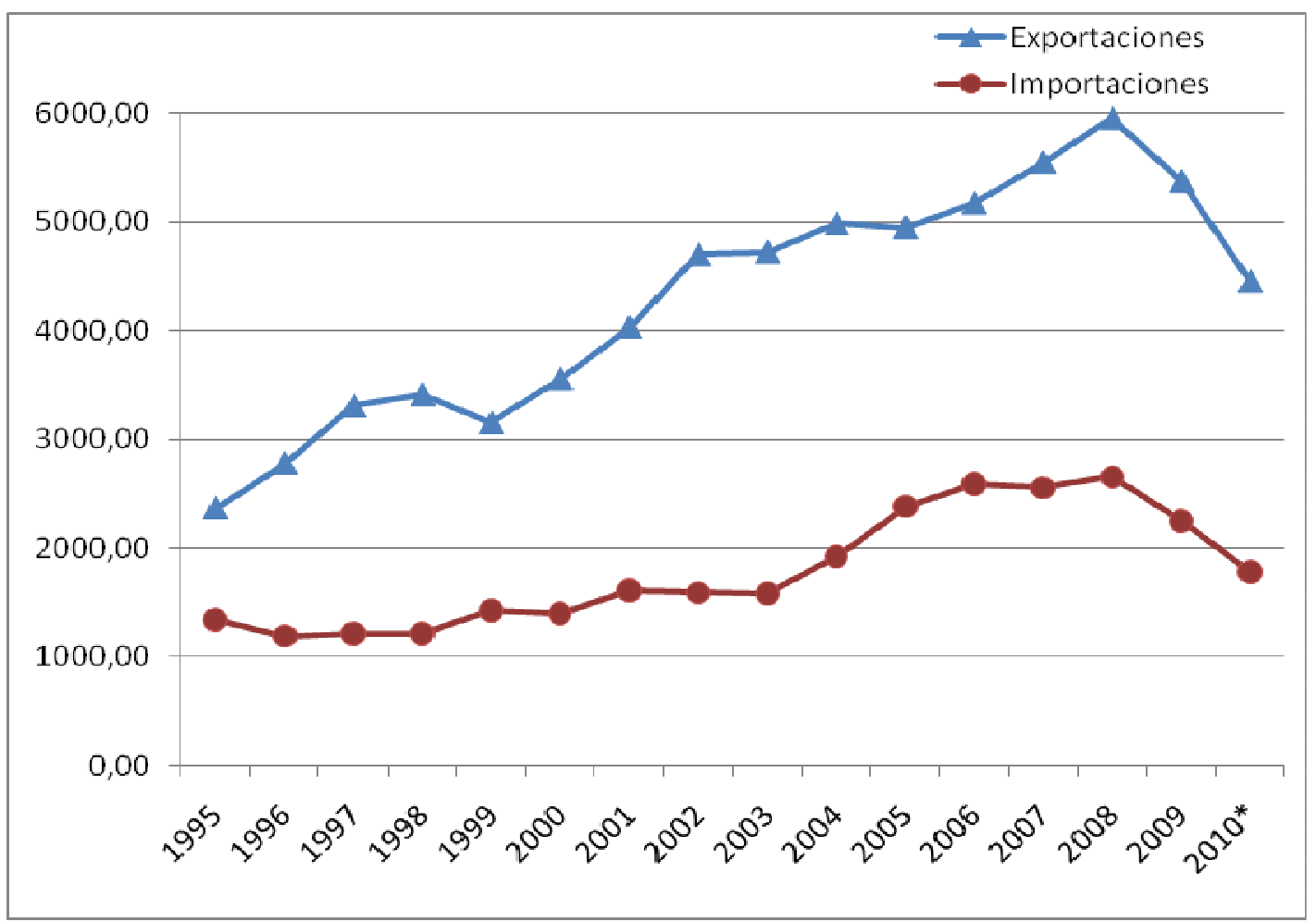

Fuente: DATACOMEX. Elaboración propia.

Si comparamos los datos de importación/exportación de alimentos de Andalucía y España se ve claramente como ésta última, aunque también tiene un balance positivo desde 1996, no tiene una situación tan favorable como la Comunidad Autónoma debido a que hay ciertas regiones en el conjunto estatal que son muy deficitarias en producción alimentaria y necesitan de las importaciones para cubrir la demanda de su mercado interno.

Todo ello a pesar de la sobreproducción de alimentos que se observa en la zona del sureste español y levante, incluyendo Andalucía, Murcia, Comunidad Valenciana y Cataluña, además de otras regiones productoras como Aragón.

\section{OFERTA Y DEMANDA}

Los últimos cambios producidos en la población española y andaluza en las últimas décadas han provocado un cambio en los hábitos de consumo agroalimentario. Así, factores como el crecimiento continuo de la población, su envejecimiento, el progresivo aumento de consumo de alimentos, la incorporación de la mujer al mercado de trabajo o el dominio de las grandes cadenas de supermercados en la oferta alimentaria, han provocado modificaciones en dicho consumo arrastrando con ellas a las empresas 
productoras a ofrecer productos más innovadores, con mayor calidad, más saludables, y más rápidos de cocinar.

\section{Figura 3. Exportación-Importación de todos los alimentos en España en el periodo 1995 - 2010 (en millones de Euros).}

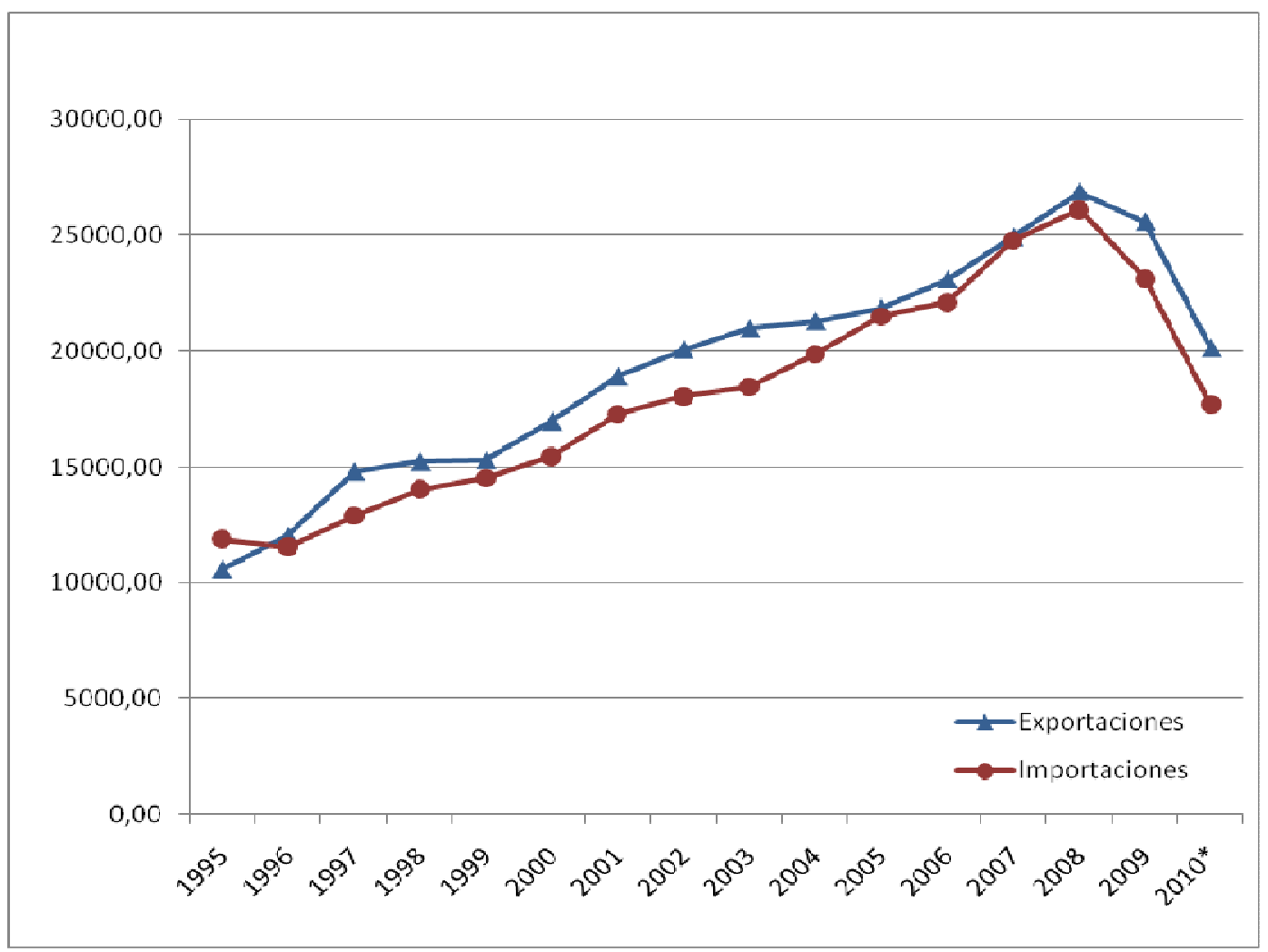

Fuente: DATACOMEX. Elaboración propia

Además, también hay que tener en cuenta que la venta de productos agroalimentarios andaluces está, como he dicho anteriormente, muy concentrada en los países de la Unión Europea $(70 \%$ del total), aunque hay otros mercados potencialmente más rentables en los que estas empresas buscan hacerse un hueco como en Estados Unidos, Japón y China, que en 2009 apenas representaban entre los tres un 5\% de las exportaciones. El resto de la producción se destina al consumo interno, tanto andaluz como español, quedando claramente reflejada la vocación exportadora de este sector.

Por otro lado, hay una característica que define perfectamente la oferta de las empresas agroalimentarias andaluzas: la atomización. Además, esta particularidad también se extiende a nivel estatal ya que el sector está compuesto en su mayoría por pequeñas y medianas empresas y, en la situación económica actual, con muchas dificultades para mantener a sus empleados y sus producciones. A ello hay que sumar también el aumento de la competencia de terceros países con producciones más rentables que cuentan con el beneplácito de la Unión Europea para introducirlas en Europa a menor precio. 
Sin embargo, del cuadro siguiente se desprende que la industria agroalimentarias a nivel español está sufriendo un ligero retroceso en cuanto a su número, aunque también es cierto que se está produciendo un fenómeno de concentración de la oferta es decir, las empresas cada vez son menos numerosas pero más grandes.

Las consecuencias de estas tendencias alcanzan no sólo a las propias empresas (mayor rentabilidad, mayor capacidad de presión a los mercados y a las instituciones, mayores producciones, etc.), sino también a los consumidores (menor oferta de productos agroalimentarios, precios pactados, etc.).

Cuadro 2. Distribución de la Industria Agroalimentaria Española

\begin{tabular}{|c|c|c|c|c|}
\hline $\boldsymbol{A N} O \boldsymbol{N}$ & $\mathbf{1}$ a 9 empleados & $\begin{array}{c}\text { 10 a 49 } \\
\text { empleados }\end{array}$ & $\begin{array}{c}\text { Más de 50 } \\
\text { empleados }\end{array}$ & TOTAL \\
\hline $\mathbf{1 9 9 9}$ & $27.923(83 \%)$ & $4.853(14 \%)$ & $1.092(3 \%)$ & 33.868 \\
\hline $\mathbf{2 0 0 7}$ & $25.255(80 \%)$ & $5.086(16 \%)$ & $1.151(4 \%)$ & 31.492 \\
\hline
\end{tabular}

Fuente.: INE. Directorio Central de Empresas. Elaboración propia.

No obstante, hay que dejar claro que el futuro del sector pasa por la innovación y la renovación de las producciones y la presentación de los productos al consumidor. Como se ve a continuación, la distribución de la oferta agroalimentaria en Andalucía está muy repartida entre a lo largo de todo su territorio. Sin embargo, por actividad, la oferta está muy concentrada en aceites y grasas vegetales (grandes extensiones de olivares), sobretodo en Córdoba y Jaén, el sector hortofrutícola, las producciones cárnicas y las enológicas y de alcoholes. Básicamente, son estos sectores los que dominan la producción agroalimentaria en Andalucía. 
Cuadro 3. Distribución de las empresas inscritas en el Registro de Industrias Agrarias de Andalucía según su actividad y por provincias. Año 2009.

\begin{tabular}{|l|c|c|c|c|c|c|c|c|c|}
\cline { 2 - 9 } \multicolumn{1}{l|}{} & Almería & Cádiz & Córdoba & Granada & Huelva & Jaén & Málaga & Sevilla & Andalucía \\
\hline Aceites y grasas vegetales & 31 & 18 & 243 & 131 & 17 & 369 & 84 & 144 & $\mathbf{1 0 3 7}$ \\
\hline Acuícolas, marisqueras y pesqueras & 6 & 81 & 7 & 10 & 36 & 8 & 31 & 37 & $\mathbf{2 1 6}$ \\
\hline Aderezos y relleno & 6 & 5 & 37 & 8 & 4 & 10 & 40 & 199 & $\mathbf{3 0 9}$ \\
\hline Aprovechamiento de fibras textiles & 0 & 0 & 5 & 0 & 0 & 1 & 0 & 17 & $\mathbf{2 3}$ \\
\hline Azúcar, mieles y ceras & 1 & 6 & 4 & 7 & 2 & 4 & 3 & 7 & $\mathbf{3 4}$ \\
\hline Cárnicas y pecuarias & 77 & 37 & 114 & 148 & 116 & 108 & 112 & 112 & $\mathbf{8 2 4}$ \\
\hline $\begin{array}{l}\text { Centrales hortofrutícolas y centros de } \\
\text { manipulación de flores y plantas }\end{array}$ & 185 & 52 & 58 & 121 & 119 & 27 & 76 & 95 & $\mathbf{7 3 3}$ \\
\hline Enológicas, alcoholes y bebidas alcohólicas & 23 & 212 & 110 & 57 & 45 & 14 & 54 & 45 & $\mathbf{5 6 0}$ \\
\hline Forestales & 3 & 2 & 3 & 4 & 7 & 4 & 5 & 9 & $\mathbf{3 7}$ \\
\hline Lácteas & 15 & 11 & 21 & 13 & 5 & 11 & 25 & 44 & $\mathbf{1 4 5}$ \\
\hline Manipulación y conservación & 13 & 14 & 106 & 31 & 5 & 85 & 14 & 17 & $\mathbf{2 8 5}$ \\
\hline $\begin{array}{l}\text { Molinería, harinas y derivados, troceados y } \\
\text { descascarados }\end{array}$ & 15 & 12 & 20 & 22 & 13 & 19 & 34 & 62 & $\mathbf{1 9 7}$ \\
\hline Otras industrias agroalimentarias & 1 & 13 & 14 & 5 & 25 & 19 & 29 & 60 & $\mathbf{1 6 6}$ \\
\hline Piensos, granos y semillas & 13 & 78 & 43 & 46 & 44 & 16 & 26 & 102 & $\mathbf{3 6 8}$ \\
\hline Servicios Técnicos & 0 & 0 & 0 & 3 & 2 & 0 & 1 & 0 & $\mathbf{6}$ \\
\hline Sín división asignada & 0 & 2 & 0 & 3 & 9 & 0 & 0 & 4 & $\mathbf{1 8}$ \\
\hline $\begin{array}{l}\text { Zumos de frutas y hortalizas, y otras } \\
\text { bebidas de carácter agroalimentario }\end{array}$ & 9 & 2 & 7 & 11 & 9 & 4 & 9 & 21 & $\mathbf{7 2}$ \\
\hline Total & $\mathbf{3 9 8}$ & $\mathbf{5 4 5}$ & $\mathbf{7 9 2}$ & $\mathbf{6 2 0}$ & $\mathbf{4 5 8}$ & $\mathbf{6 9 9}$ & $\mathbf{5 4 3}$ & $\mathbf{9 7 5}$ & $\mathbf{5 0 3 0}$ \\
\hline
\end{tabular}

Fuente: Junta de Andalucía. Consejería de Agricultura y Pesca. Elaboración propia. 
Según la Consejería de Agricultura y Pesca de la Junta de Andalucía, el número de empresas agroalimentarias en 2007 era de 8.578, de los que el 45\% corresponden a establecimientos de elaboración de pan, galletas y productos de panadería y pastelería, empleando muchas de ellas a menos de 5 personas. Por ello se puede deducir que un gran número de empresas en varios de estos sectores como el de las panaderías no son más que "pequeñas industrias" con pocos o ningún trabajador contratado y poco volumen de facturación y, por tanto, sin la obligatoriedad de inscribirse en el Registro de Industrias Agrarias de Andalucía como tales.

En resumen se puede decir que factores externos al sector agroindustrial como la situación económica actual o los cambios en la demanda de sus productos y factores internos como la alta atomización o su creciente concentración han dado como resultado que desde principios del siglo XXI, este sector haya experimentado un descenso notable en el número de industrias agroalimentarias en Andalucía, provocando el descenso del número de empleados y fortaleciendo a las empresas más grandes.

Figura 4. Evolución de las empresas del sector agroalimentario (2000-2009).

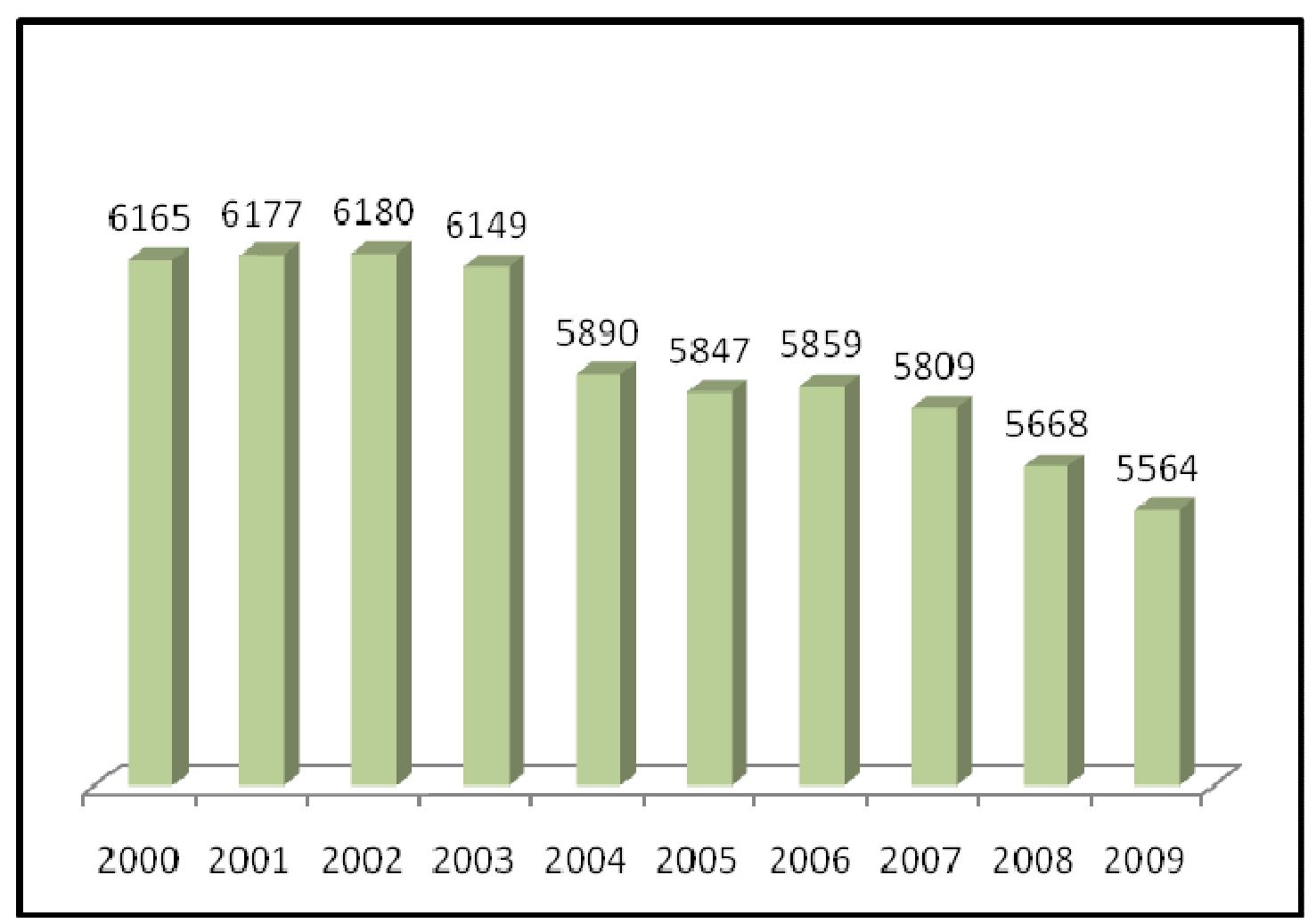

Fuente: Junta de Andalucía. Análisis del contexto del sector agroalimentario en Andalucía. Sevilla.2009.

\section{LA GRAN DISTRIBUCIÓN Y EL COOPERATIVISMO EN EL SECTOR AGROALIMENTARIO}

En Andalucía, las empresas de distribución son en la actualidad el factor que más incide en la cadena agroalimentaria. Basada en dos principios básicos de localización: proximidad a las materias primas y proximidad a los mercados finales, se ha convertido en una red de unas pocas 
empresas de gran tamaño que manipulan y configuran a su antojo a productores y consumidores.

Según el Plan Estratégico para la Agroindustria Andaluza. Horizonte 2013, en los últimos años también se está dando un proceso de concentración empresarial en la distribución, haciendo más evidentes sus características principales:

- Trabaja con grandes volúmenes y márgenes estrechos.

- Se trata de un negocio financiero que impone ingresos al contado pero instaura el pago diferido a los proveedores.

- Trabaja con surtidos muy amplios, conformados por una gran cantidad de categorías, referencias y formatos.

- Gestiona la venta de muchos productos perecederos que requieren un tratamiento especial.

Todo este poder concentrado por la gran distribución va en aumento día a día por lo que, es de suponer, en un futuro la tendencia sea la misma. Además, estas grandes empresas se ven reforzadas por la venta de sus marcas blancas, más asequibles y que les confieren todavía más capacidad de maniobra frente a sus proveedores.

Para hacer frente a todo este proceso sería conveniente que las empresas agroindustriales fomentaran la concentración empresarial a través de fórmulas como el asociacionismo o el cooperativismo con el fin de no estar sujetos a los caprichos y deseos de estas grandes distribuidoras que aumentan su beneficio a costa de mermar el de los productores y de subir el precio a los consumidores.

El objetivo final de estos procesos es la integración vertical de las empresas agroalimentarias en la distribución. No obstante, hay que tener en cuenta que la actual situación económica no ofrece perspectivas muy positivas para nuevas inversiones y aventuras financieras por lo que la creación de cooperativas en esta rama está algo paralizada en Andalucía. Además, las que existen, según fuentes de la Federación Andaluza de Empresas Cooperativas Agrarias (Faeca), han registrado durante 2010 un descenso de un $2 \%$ en sus ingresos.

Dejando a un lado el aspecto económico, las motivaciones que llevan a la formación de cooperativas es diversa y, en general, los factores que influyen en su constitución son los siguientes (Cervera y Bohórquez, 2000):

- Toma de decisiones excluyentes que sopesen las ventajas e inconvenientes del proyecto.

- Inversión inicial relativa por las características únicas de cada agricultor.

- El tamaño de la cooperativa puede ser atrayente o no para determinados agricultores.

- Volumen de producción aportado al proyecto diferente para cada agricultor.

- Homogeneidad de la base social.

- Exclusividad de las producciones de los socios hacia su cooperativa. 
- Existencia de intereses externos a la creación de la cooperativa como subvenciones, intereses políticos, etc, que pueden hacer perder la independencia de la misma.

No obstante, a pesar de todos estos factores no hay que obviar que las cooperativas agroalimentarias también poseen aspectos negativos.

Cuadro 4. Debilidades y fortalezas de las cooperativas agroalimentarias andaluzas.

\begin{tabular}{|c|c|}
\hline Debilidades & Fortalezas \\
\hline $\begin{array}{c}\text { La población agraria disminuye y } \\
\text { envejece }\end{array}$ & $\begin{array}{c}\text { La mayoría de los agricultores son socios } \\
\text { de alguna cooperativa }\end{array}$ \\
\hline $\begin{array}{c}\text { La superficie agrícola disminuye } \\
\text { capitalizadas cooperativas no están suficientemente }\end{array}$ & $\begin{array}{c}\text { Las cooperativas recogen buena parte de } \\
\text { la producción agroalimentaria }\end{array}$ \\
\hline $\begin{array}{c}\text { Insuficiente orientación al mercado } \\
\text { suele ser muy alta }\end{array}$ & $\begin{array}{c}\text { Apoyo de las Administraciones a las } \\
\text { cooperativas por sus problemas de } \\
\text { competitividad }\end{array}$ \\
\hline $\begin{array}{c}\text { Insuficiente productividad de las } \\
\text { cooperativas }\end{array}$ & \\
\hline
\end{tabular}

Fuente: Elaboración propia a partir de Juliá Igual J.F. y Fernández Méndez M. (1998).

Como se puede ver en este cuadro, problemas como el envejecimiento o la pérdida de suelo agrícola están presentes no solo en el propio sector agroindustrial sino también en todo el ámbito agrario andaluz y español. Además, muchas de estas cooperativas no generan interés o tienen producciones insuficientes y no pueden obtener el capital necesario para su mantenimiento. Sin embargo, también hay que destacar el apoyo institucional hacia estas agrupaciones y, sobretodo, el hecho de que la mayoría de los agricultores formen parte de alguna cooperativa.

\section{CALIDAD Y DENOMINACIÓN DE ORIGEN DE LOS PRODUCTOS}

La calidad de un producto en comparación con otros (calidad diferencial) viene dada por una serie de características por las que se le reconoce. Éstas están relacionadas con sus propiedades, origen, forma, color, textura, método de elaboración, etc, y, cada vez más, el consumidor final tiene interés por conocerlas y tener claro qué están comiendo.

En Andalucía, muchos son los productos que gozan de prestigio tanto en el mercado nacional como en el internacional, derivado de aspectos como su elaboración (por ejemplo el aceite), zona de producción (como jamones y embutidos), etc.

Dentro de las protecciones y reconocimientos que se otorgan a estos productos encontramos los siguientes (Junta de Andalucía, 2009): 
1. Denominación de Origen Protegida (DOP). Da cobertura a productos cuya producción, transformación y elaboración debe realizarse en una zona geográfica determinada y con unos procedimientos específicos.

2. Indicación Geográfica Protegida (IGP). Con un estrecho vínculo entre el medio geográfico y el producto.

3. Especialidad Tradicional Garantizada (ETG). Esta certificación no hace referencia al origen sino que destaca la composición del producto o su elaboración tradicional.

4. Indicación de Agricultura Ecológica. Certificación a aquellos productos agrarios obtenidos a través de procesos productivos respetuosos con el medio en todas sus fases.

5. Producción Integrada. Hace referencia a productos agrícolas, ganaderos y transformados obtenidos de una agricultura que trata de racionalizar el empleo de insumos en base a criterios ambientales, técnicos y económicos. Hay que destacar que Andalucía ha sido pionera en la regulación de estas producciones a través del Decreto 215/1995 y del Decreto 245/2003.

Por otro lado, además de los certificados y normativas estatales y europeas, la Junta de Andalucía participa en el concepto de calidad otorgando la marca de Calidad Certificada, aplicada a los productos acogidos a alguna de las denominaciones anteriores. El uso de esta marca se concede en periodos de cinco años renovables y cuya adscripción es gratuita. También hay numerosos productos andaluces de esta rama que se acogen a protecciones de calidad a nivel estatal tales como la Calidad Total, que abarca toda la actividad de las empresas y que está concedida por organismos independientes como AENOR o SGS.

Por último, también existen otras certificaciones como la Seguridad Alimentaria, que es la garantía de la seguridad de los productos alimenticios que se consumen. Está basada principalmente en la prevención y en el análisis de los productos antes de que lleguen a los mercados. Además, está garantía tiene que estar certificada en primer lugar por la empresa productora $\mathrm{y}$, en segundo, por organismos independientes y públicos de sanidad.

Según el Libro Blanco sobre Seguridad Alimentaria, estas políticas de prevención deben basarse en un planteamiento global e integrado a lo largo de toda la cadena alimentaria, entre todos los Estados Miembros y entre los países vinculados con la Unión Europea.

El fin último de todas estas normas y certificaciones no es otro que garantizar al consumidor final la máxima calidad de los productos además de ofrecerles alimentos con determinadas características organolépticas, libres de peligros y cada vez más variados. Por ello, las empresas productoras han de ofrecer sus propios mecanismos de trazabilidad y autocontrol, además del complemento que suponen las instituciones y organismos que garantizan la calidad de estas producciones fuera de estas empresas, por un lado, y por otro, que estos mecanismos descritos funcionen correctamente. 


\section{EXPORTACIONES DEL SECTOR AGROALIMENTARIO ANDALUZ}

Es evidente que la agroindustria andaluza tiene una clara vocación exportadora, sobre todo a mercados europeos. Esta característica queda aún más patente si hablamos de cifras económicas. En 2008, estas exportaciones supusieron 5.947 millones de euros, es decir el 35\% del total de las exportaciones andaluzas. Además, respecto a 2007, éstas aumentaron un $7 \%$. Sin embargo, hay que aclarar que este signo positivo deriva de los magníficos números que registran los subsectores de frutas y hortalizas y de aceites y grasas, con un saldo comercial muy positivo que compensan el resto de subsectores. A modo de ejemplo, en 2008, ambos obtuvieron un saldo favorable de 2.847 millones de euros.

La importancia de este sector en Andalucía no sólo viene dada por las cifras dadas hasta ahora sino que además representa para la región el futuro para la agricultura ya que necesita de una gran cantidad de materias primas. Por otro lado, ejerce un efecto de arrastre sobre otras actividades económicas auxiliares como la logística o la fabricación y reparación de maquinaria y supone un nexo de unión en el entorno rural puesto que es el entorno mayoritario donde se localizan estos establecimientos.

Las innovaciones y la modernización experimentadas en este sector para hacer frente a los nuevos mercados y a las nuevas demandas de los consumidores se han venido produciendo desde los años ochenta del siglo pasado a través de las ayudas ofrecidas desde el ámbito comunitario y también desde el estatal. Estas ayudas, en el periodo 2000-2007 supusieron un 30\% del total invertido (600 millones de euros de los 2000 millones invertidos en total).

Según el diagnóstico elaborado por el Plan Estratégico para la Agroindustria andaluza. Horizonte 2013, el balance exterior del sector en la región es el siguiente en 2008, respecto a 2007 :

- Las exportaciones crecieron un 7\%

- Las importaciones solo aumentaron un 3\%

- El saldo comercial creció favorablemente un $11 \%$

A esto hay que añadir que las exportaciones andaluzas de todos los alimentos supusieron un $35 \%$ del total de las exportaciones de la Comunidad Autónoma y que la tasa de cobertura de alimentos en 2008 alcanzó el $226 \%$.

Dentro de este sector, las tres principales ramas exportadoras son las siguientes:

- Frutas y legumbres. Representan el 20\% del total de las exportaciones andaluzas y el $40 \%$ de las exportaciones del sector en España, siendo Almería la provincia más importante en su producción.

- Grasas y aceites. Representan el 8\% de las exportaciones andaluzas y el 53\% de las exportaciones del sector en España. 
- Bebidas. Representan el 1,5\% de las exportaciones de Andalucía y el 9\% de las exportaciones del sector en España.

La evolución de las exportaciones del sector agroalimentario en Andalucía ha venido marcada, en los últimos años, por un crecimiento continuo a pesar de la coyuntura económica y la saturación de los mercados maduros. Las tres ramas del sector descritas arriba supusieron en 2008 el 83,7\% del total de las exportaciones agroalimentarias lo que da una idea de la importancia y magnitud de los datos manejados. A ellos hay que sumar que otros productos, aunque con unos valores absolutos menores, porcentualmente también crecieron sobremanera. Tal es el caso del azúcar, del café y del cacao, que crecieron más de un $98 \%$ en 2008 o del tabaco, que creció un 74,8\%.

No obstante, para que el sector siga creciendo en un futuro no sólo basta con revisar las cuentas de años anteriores. El principal objetivo en estos momentos es el de buscar nuevos mercados con nuevos consumidores y menos competencia. Por ello la Agencia Andaluza de Promoción Exterior (Extenda) organizó en 2010 la Semana de Andalucía en el Pabellón de España, dentro de la Exposición Universal de Shanghái.

El objetivo principal de esa expedición fue el de facilitar la introducción de las marcas y Denominaciones de Origen andaluzas en el mercado chino además de contactar con importadores, distribuidores y cadenas de supermercados del gigante asiático. Según datos del ICEX (Instituto Español de Comercio Exterior), las empresas que exportaron hacia China en 2009 fueron un $25 \%$ más que en 2008, lo que sigue corroborando la importancia del sector para la economía española y andaluza.

Como no podía ser de otro modo, uno de los productos más valorados por los asiáticos es el aceite de oliva, que ha multiplicado por seis el valor de sus exportaciones en los últimos cuatro años. Este fenómeno se ha producido en parte por la campaña que la Junta de Andalucía puso en marcha en 2006 con el objetivo de promocionar el producto y de dar a conocer sus propiedades.

Todo este proceso de marketing ha supuesto que en 2009 Andalucía exportara aceite a China por un valor de 14,5 millones de euros, lo que supuso un incremento del $50 \%$ respecto a 2008. En resumen, Andalucía ha alcanzado el liderazgo mundial del aceite de oliva en China, acaparando una cuota de mercado en el gigante asiático del 39\%, casi diez puntos por encima de otros competidores como Italia.

\section{CONCLUSIÓN}

Como se ha podido ver, el sector tiene una importancia capital en Andalucía desde un punto de vista económico y social ya que son muchos los trabajos que genera este conjunto de actividades que además, en su mayoría, se desarrollan en el ámbito rural.

Sin embargo, y a pesar de todos estos buenos números, hay que seguir invirtiendo e innovando en nuevos productos y nuevas maneras de producir y elaborar y, por otro lado, también se hace necesario estudiar las demandas de los mercados, siempre cambiantes, y adentrarse en otros nuevos, potencialmente más dinámicos. Por ello, el Plan Estratégico para la Agroindustria Andaluza. Horizonte 2013, ha recomendado el 
cumplimiento de unos objetivos fundamentales dentro del ámbito de los mercados exteriores:

- Capitalizar las fortalezas sectoriales existentes en Andalucía

- Orientar esfuerzos al desarrollo de una nueva oferta sectorial con potencial exterior y capacidades internas

Además de los objetivos marcados en dicho informe, es conveniente también tener en cuenta que la madurez de los mercados europeos, la intensidad de la competencia existente en los mismos y los crecimientos económicos que se están dando en otras áreas geográficas con potencial para exportar producciones similares, aconsejan diversificar progresivamente los destinos de las producciones andaluzas principalmente a Asia, pero sin perder de vista mercados en los que estos productos apenas tienen representaciones como los Estados Unidos.

Por tanto, y en relación con las exportaciones e inversiones necesarias en el sector, hay que tener claros los retos a los que se enfrenta el sector en el futuro más inmediato:

- Aceleración del proceso de globalización

- Fuerte concentración de la distribución

- Competencia global y oportunidades de acceso a mercados exteriores

- Nuevos perfiles de consumidores

Todo ello puede suponer debilidades, pero también oportunidades de expansión y de crecimiento para las empresas agroalimentarias que sean capaces de seguir innovando e investigando para cubrir nichos de mercado o adentrarse en áreas geográficas en las que estos productos no estén aún presentes.

Para llevar a cabo todo este proceso, la gestión global, las TICs, la calidad diferenciada, las economías de escala, la mayor preocupación ambiental y la elaboración de productos con mayor valor añadido, entre otros aspectos, son fundamentales para seguir, sino creciendo económicamente, sí al menos subsistir en un mundo en que las grandes empresas productoras y distribuidoras concentran cada vez más cuota de mercado. Además, también hay que tener en cuenta hacia dónde se dirige el mercado y las nuevas demandas para poder hacerles frentes a través de la creación de nuevas marcas blancas, aumentando la capacidad de negociación de las grandes empresas con marcas más consolidadas, facilitando la apertura de nuevos mercados y recordando la competencia que suponen los países emergentes.

En resumen, incluso en un entorno tan globalizado como el actual, con nuevos competidores y bajos costes, la agricultura y la agroindustria andaluzas son competitivas, aportando valor diferencial con su oferta para conseguir que este sector sea el motor del nuevo modelo productivo andaluz. 


\section{BIBLIOGRAFÍA}

AGUILAR GÓMEZ, J. La industria agroalimentaria en Andalucía. Cuadernos, 1995, ${ }^{\circ}$ 28. p. 55-62.

CERVERA PAZ, A. y BOHÓRQUEZ BOTANA, M. El asociacionismo como fuente de ventaja en el sector agroalimentario andaluz. Revesco. Revista de Estudios Cooperativos, 2000, $\mathrm{n}^{\mathrm{o}} 70$.

Instituto de Estudios Cajamar. El complejo agroalimentario andaluz. Presente y Futuro. Almería, 2005.

Empresa Pública de Desarrollo Agrario y Pesquero, Consejería de Agricultura y Pesca. Plan Estratégico de la Agroindustria Andaluza. Sevilla, 2002.

JULIÁ IGUAL J. F. y FERNÁNDEZ MENDEZ M. Concentracion empresarial, Internacionalizacion. Actuaciones del Cooperativismo agrario en la Unión Europea. Documentos, 1998, n 12.

Junta de Andalucía (Consejería de Agricultura y Pesca). Modernización e Industria Agroalimentaria Andaluza. Sevilla, 2006.

Junta de Andalucía (Consejería de Agricultura y Pesca). Análisis del contexto del sector agroalimentario en Andalucía. Sevilla, 2009.

Junta de Andalucía (Consejería de Agricultura y Pesca). Plan Estratégico para la Agroindustria Andaluza. Horizonte 2013. Sevilla, 2009.

MORALES GIL, A. Aspectos geográficos de la horticultura de ciclo manipulado en España. Universidad de Alicante, 1997. p. 17-31.

RUIZ MOLINA, A. Estrategias para el desarrollo de la agroindustria en Andalucía. Especial referencia al sector de transformados vegetales. I Congreso de Ciencia Regional de Andalucía: Andalucía en el umbral del siglo XXI. Jerez, 1997.

\section{RECURSOS ELECTRÓNICOS}

Base estadistica de la Junta de Andalucía [en línea].

$<$ http://www.juntadeandalucia.es:9002/anuario/index2.htm> [Consulta: 20 junio 2011].

Andalucía cuenta ya con 21 empresas agroalimentarias con distintivo "Calidad Certificada" [en línea]. 20 Diciembre 2002. <http://www.consumer.es/seguridadalimentaria/2002/12/20/4512.php> [Consulta: 22 junio 2011].

Andalucía impulsa una red de parques tecnoalimentarios [en línea]. 28 Noviembre 2003. < http://www.consumer.es/seguridad-alimentaria/2003/11/28/9621.php> [Consulta: 21 junio 2011].

El reto de la innovación se implanta en la agroindustria [en línea]. 28 Julio 2009. $<\mathrm{http} / /$ www.andaluciainformacion.es/portada/?a=60877\&i=8\&f=0\&b=200006> [Consulta: 25 junio 2011]. 
Extenda muestra en Shanghai la agroindustria andaluza [en línea]. 01 Agosto 2010. $<$ http://www.europasur.es/article/economia/759791/extenda/muestra/shanghai/la/agroin dustria/andaluza.html> [Consulta: 21 junio 2011].

La Consejería apuesta por China [en línea]. 01 Septiembre 2010.

$<$ http://www.andaluciaunica.com/noticia/6037/PROMOCI\%C3\%93N/consejer\%C3\%A Da-apuesta-china.html> [Consulta: 22 junio 2011].

El Gobierno andaluz resalta que la Ley de Calidad Alimentaria reforzará al sector y perseguirá el fraude' [en línea]. 19 Octubre 2010.

$<$ http://www.efeagro.com/MicrositeHome.aspx?idmicrosite=20\&idcategoria=16\&idmul timedia=1387404> [Consulta: 21 junio 2011]. 